\title{
Phase Transitions in the Hexagonal Tungsten Bronze $\mathrm{RbNbW}_{2} \mathrm{O}_{9}$
}

\author{
Jason A. McNulty ${ }^{1}$, Alexandra S. Gibbs ${ }^{2}$, Philip Lightfoot ${ }^{1}$ and Finlay D. Morrison ${ }^{1 *}$
}

${ }^{1}$ EaStCHEM, School of Chemistry, University of St Andrews, St Andrews, KY16 9ST, United Kingdom

${ }^{2}$ ISIS Facility, Rutherford Appleton Laboratory, Chilton, Oxon, OX11 0QX, United Kingdom

\begin{abstract}
The hexagonal tungsten bronze $\mathrm{RbNbW}_{2} \mathrm{O}_{9}$ is shown, by variable-temperature powder neutron diffraction and symmetry-mode analysis, to display a significantly different phase transition sequence compared to the related $\mathrm{CsNbW}_{2} \mathrm{O}_{9}$ composition. At ambient temperature, $\mathrm{RbNbW}_{2} \mathrm{O}_{9}$ adopts the polar orthorhombic space group $\mathrm{Cmc} 2_{1}$. Upon heating, the thermal evolution of the crystal structure proceeds via two transitions. These correspond to sequential loss of two distinct octahedral tilting modes, leading to space group $P 6_{3} m c$ at around $655 \mathrm{~K}$, and space group $P 6 \mathrm{~mm}$ near $700 \mathrm{~K}$. The polar distortion is retained up to the highest temperature studied here. The differences in structural behaviour between the proper ferroelectric $\mathrm{RbNbW}_{2} \mathrm{O}_{9}$ and the improper ferroelectric $\mathrm{CsNbW}_{2} \mathrm{O}_{9}$ emphasises the need for careful crystallographic analyses of materials of this type.
\end{abstract}

Keywords: Powder neutron diffraction; Symmetry-mode analysis; Phase transitions; Hexagonal tungsten bronze; Proper ferroelectricity

\section{Introduction}

Noncentrosymmetric materials have been studied extensively due to their wide range of symmetry-dependent properties and the pursuit of tuneable structural and electrical behaviour.[1] The perovskite and tetragonal tungsten bronze (TTB) families have undergone extensive studies due to their compositional flexibility[2-5] but a related family of materials, the hexagonal tungsten bronzes (HTBs), have only recently begun to garner similar interest. This structure type is derived from the hexagonal polymorph of tungsten trioxide $\left(h-\mathrm{WO}_{3}\right)$ and consists of corner-linked $\mathrm{BO}_{6}$ octahedra forming hexagonal and trigonal channels down the $c$ axis. The unit cell of the aristotype crystal structure has space group P6/mmm with $a_{0} \sim 7.4, c_{0}$ $\sim 4.0 \AA$. This structure can also be stabilised by intercalation of a cation into the hexagonal channel resulting in the general formula $\mathrm{A}_{x} \mathrm{BO}_{3}(0.15 \leq x \leq 0.33)$, where $\mathrm{A}$ is usually a large 
group 1 metal such as $\mathrm{K}^{+}, \mathrm{Rb}^{+}$and $\mathrm{Cs}^{+}$and $\mathrm{B}$ is usually $\mathrm{W}^{\mathrm{V}} / \mathrm{W}^{\mathrm{VI}}$.[6] These materials often exhibit metallic and conductive behaviour, however this can be reduced by replacement of $\mathrm{W}^{\mathrm{V}}$ with $d^{0}$ cations, resulting in the formula $A B \mathrm{~W}_{2} \mathrm{O}_{9}$. Although this type of compositional manipulation has been studied extensively,[7] there are few examples of the crystal structure being either assigned unambiguously or related to physical property measurements. The most recent examples of these materials have focused on the structures of the $A \mathrm{NbW}_{2} \mathrm{O}_{9}$ series with $A=\mathrm{K}^{+}, \mathrm{Rb}^{+}$and $\mathrm{Cs}^{+} .[8-10]$ Investigations into the structure-property relationships of $\mathrm{CsNbW}_{2} \mathrm{O}_{9}$ have shown this material to be an electronically-driven improper ferroelectric with an interesting domain microstructure that may potentially be useful in domain wall nanoelectronics.[9] Although neither $\mathrm{RbNbW}_{2} \mathrm{O}_{9}$ nor $\mathrm{KNbW}_{2} \mathrm{O}_{9}$ appear to be improper ferroelectrics, recent work involving detailed crystallographic characterisation of the ambient temperature structures utilising high-resolution powder neutron diffraction (PND) has shown that both compositions feature octahedral tilting at low temperature, as also observed in $\mathrm{CsNbW}_{2} \mathrm{O}_{9}$. This leads to a doubling of the $c$-axis, resulting in orthorhombic space group $C m c 2_{1}\left(a_{\mathrm{O}} \sim a_{0}, b_{\mathrm{O}} \sim \sqrt{ } 3 a_{0}, c_{\mathrm{O}} \sim 2 c_{0}\right)$.[10]

We now report the first variable temperature study of $\mathrm{RbNbW}_{2} \mathrm{O}_{9}$ using high-resolution PND. In particular, we use both symmetry mode analysis and physical property measurements to explore systematically the underlying mechanisms of symmetry-breaking.

\section{Experimental}

\subsection{Synthesis}

Samples were prepared via traditional solid-state synthesis using the methodology presented in our previous work.[10] Sacrificial powder, comprised of the unreacted reagents, was used to cover the pellets to minimise alkali metal loss. Pellets for electrical measurements were mixed with a small amount of polyvinyl alcohol (PVA) solution to act as a binding agent and pressed under $200 \mathrm{MPa}$ using an isostatic oil press. The pellet was sintered at $1223 \mathrm{~K}$ for $1 \mathrm{~h}$ and subsequently polished using fine-grained SiC paper (P800). Ceramic pellet density was relatively low (70-80\% of the theoretical density). SEM images of the surface of ceramic $\mathrm{RbNbW}_{2} \mathrm{O}_{9}$ are shown in the ESI. After polishing, sputtered gold electrodes were applied to the opposing pellet faces and capped with silver conductive paste which was subsequently cured at $150{ }^{\circ} \mathrm{C}$ for 20 mins to provide protection at high temperature. 


\subsection{Powder Diffraction}

Preliminary X-ray diffraction data was collected using a PANalytical EMPYREAN diffractometer with a $\mathrm{Cu} \mathrm{K}_{\alpha}$ source to confirm sample purity. Powder neutron diffraction (PND) data were collected on the high-resolution powder diffractometer (HRPD) at the ISIS spallation neutron source using the time-of-flight method. Data were collected from 288 to $1173 \mathrm{~K}$ with scans recorded for detector currents of $40 \mu \mathrm{Ah}$ integrated proton current to the target (approximately $1 \mathrm{~h}$ of continuous beam). Powdered samples were contained in a cylindrical vanadium can. For analysis, two detector banks covering $d$-spacing ranges of $0.7<$ $d<2.6$ and $0.9<d<4.0 \AA$, respectively, were used.

Data were analysed by the Rietveld method, using the GSAS/EXPGUI package[11,12] and interpretation of the results assisted using symmetry mode analysis via ISODISTORT.[13] A consistent refinement strategy was used in each case with a 12-term shifted Chebyschev background function used to account for background coefficients. Parameters refined were lattice parameters, atomic positions, isotropic atomic displacement parameters, appropriate diffractometer constants and profile coefficients. Small peaks from the vanadium can were identified in PND patterns; these were not included in the refinements.

\subsection{Dielectric Data}

Dielectric data were collected at intervals of $1 \mathrm{~K}$ with heating/cooling rates of $2 \mathrm{~K} \mathrm{~min}^{-1}$ using a Wayne Kerr 6500B impedance analyser with the sample mounted in a tube furnace. Data were collected over the frequency range of $20 \mathrm{~Hz}$ to $10 \mathrm{MHz}$ with an ac amplitude of $500 \mathrm{mV}$.

\section{Results and Discussion}

\subsection{Thermal evolution of the crystal structure}

In our previous work we have shown that the ambient temperature structure is orthorhombic, space group $C m c 2_{1}(a \sim 7.3, b \sim 12.7, c \sim 7.8 \AA)$.[10] A minor pyrochlore impurity phase $\left(\mathrm{RbNbWO}_{6}, \sim 1\right.$ wt. \%) is present, however this was not included in refinements due to the minimal contribution to the observed diffraction pattern. 
Due to the relative insensitivity of lattice parameters to the exact space group, preliminary Rietveld refinements were carried out in $C m c 2_{1}$ over the entire temperature range as a means of investigating possible transition temperatures (Fig. S1).

A change in the gradient of the $c$-axis thermal expansion is observed between 673 and $723 \mathrm{~K}$, similar to that observed in the related $\mathrm{CsNbW}_{2} \mathrm{O}_{9}(\sim 355 \mathrm{~K})$ system. In $\mathrm{CsNbW}_{2} \mathrm{O}_{9}$, this corresponds to the loss of octahedral tilting and consequently the loss of $c$-axis doubling. Above $973 \mathrm{~K}$, a decrease in the $c$-parameter is observed suggestive of the transition from the polar to the paraelectric phase $(P 6 / \mathrm{mmm})$ based on the analogous behaviour observed in $\mathrm{CsNbW}_{2} \mathrm{O}_{9}$. Additionally, an apparent convergence of the $a$ - and $b$-parameters occurs above $500 \mathrm{~K}$, suggesting the transition to a hexagonal space group. There is no evidence of an abrupt change in the lattice parameters, volume, or in the permittivity and dielectric loss of the material (Fig. S3).

From the group-subgroup relationship, transition from the ambient temperature $C m c 2_{1}$ structure may proceed via either orthorhombic $(\mathrm{Cmm} 2$ or $\mathrm{Cmcm})$ or hexagonal $\left(\mathrm{P}_{3} \mathrm{~cm}\right.$ or $\mathrm{P}_{3} m c$ ) space groups. The orthorhombic $\mathrm{Cmcm}$ structure and both hexagonal structures feature octahedral tilting, as observed in $C m c 2_{1}$. Our previous work showed that the octahedrally tilted $C m c 2_{1}$ structures in both $\mathrm{RbNbW}_{2} \mathrm{O}_{9}[10]$ and $\mathrm{CsNbW}_{2} \mathrm{O}_{9}[9]$ are described by inclusion of $\mathrm{A}_{6}{ }^{+}$ and $\mathrm{A}_{3}{ }^{+}$distortion modes, consistent with previous work.[14-16] In contrast to $\mathrm{CsNbW}_{2} \mathrm{O}_{9}$, which has a more complex supercell at ambient temperature due to an additional $\mathrm{K}_{3}$ distortion mode, the structural evolution of $\mathrm{RbNbW}_{2} \mathrm{O}_{9}$ is unlikely to proceed via $\mathrm{P}_{3} \mathrm{~cm}$ as this requires an $\mathrm{A}_{4}{ }^{+}$mode, which has previously been shown by Whittle et al. [14] to result in disconnection of the $\mathrm{BO}_{6}$ octahedral framework.

Comparison of the goodness-of-fit parameters obtained from Rietveld refinement at $673 \mathrm{~K}$ indicates that the $\mathrm{P}_{3} m c$ model results in a better fit than any of the other possibilities (Table 1). The assignment of this hexagonal space group is supported by observation of additional peaks due to the orthorhombic distortion that are lost with increasing temperature (Fig. 1a) as well as the previously discussed convergence of the $a$ - and $b$-parameters in the preliminary refinements (Fig. S1). The transformation from $C m c 2_{1}$ to $P 6_{3} m c$, while allowed by the groupsubgroup relationship, must be a first-order transition and therefore discontinuity in both the unit cell volume and dielectric data would be expected. The crystallographic details for the $P 6_{3} m c$ model at $673 \mathrm{~K}$ are presented in Table 2 and the corresponding Rietveld fit in Fig. 2a. The change in unit cell size from the orthorhombic to hexagonal phase is shown in Fig. 3. 
Table 1. Comparison of the respective Rietveld refinements of PND data obtained at $673 \mathrm{~K}$ for $\mathrm{RbNbW}_{2} \mathrm{O}_{9}$ refined in the feasible crystallographic models. Unit cell metrics are shown with respect to the parent aristotype $P 6 / \mathrm{mmm}$ structure with parameters $a_{0}$ and $c_{0}$. The total number of refineable parameters in each case is given by $N_{\text {tot }}$.

\begin{tabular}{llllll}
\hline Model & Metrics & $\chi^{2}$ & $\mathrm{w} R p(\%)$ & $R p(\%)$ & $N_{\text {tot }}$ \\
\hline$C m c 2_{1}$ & $a_{0} \times \sqrt{ } 3 a_{0} \times 2 c_{0}$ & 6.634 & 4.67 & 3.94 & 63 \\
$C m c m$ & $a_{0} \times \sqrt{ } 3 a_{0} \times 2 c_{0}$ & 9.314 & 5.54 & 4.56 & 54 \\
$P 6_{3} m c$ & $a_{0} \times a_{0} \times 2 c_{0}$ & 5.932 & 4.42 & 3.76 & 51 \\
$P 6_{3} c m$ & $a_{0} \times a_{0} \times 2 c_{0}$ & 7.375 & 4.93 & 3.98 & 49 \\
$C m m 2$ & $a_{0} \times \sqrt{ } 3 a_{0} \times c_{0}$ & 7.849 & 5.08 & 4.06 & 55 \\
\hline
\end{tabular}
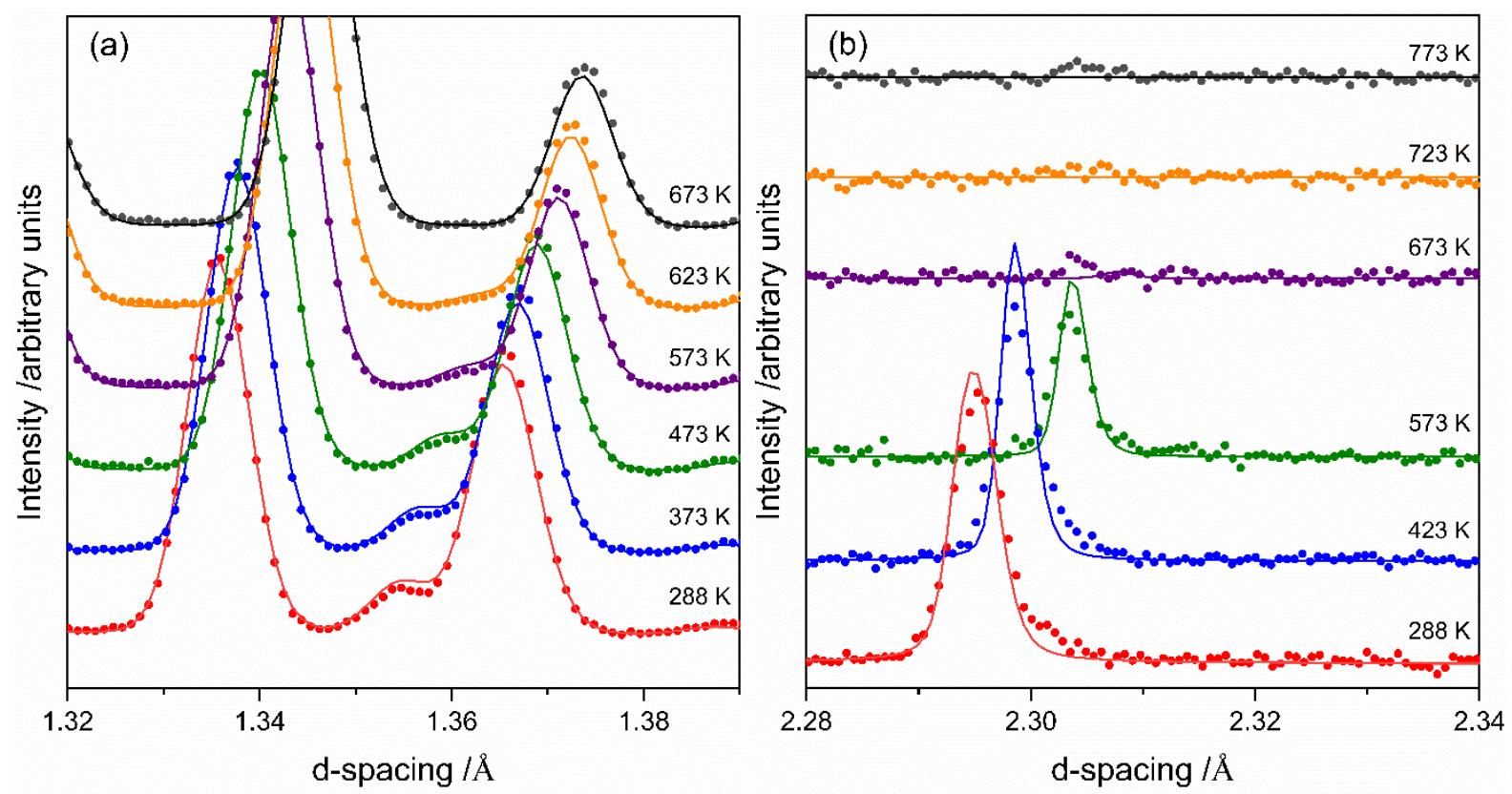

Fig. 1. Structural evolution as a function of increasing temperature indicating loss of reflections associated with a) an orthorhombic distortion and b) $c$-axis doubling due to octahedral tilting. Filled circles represent data points and the solid lines are the Rietveld fits. 

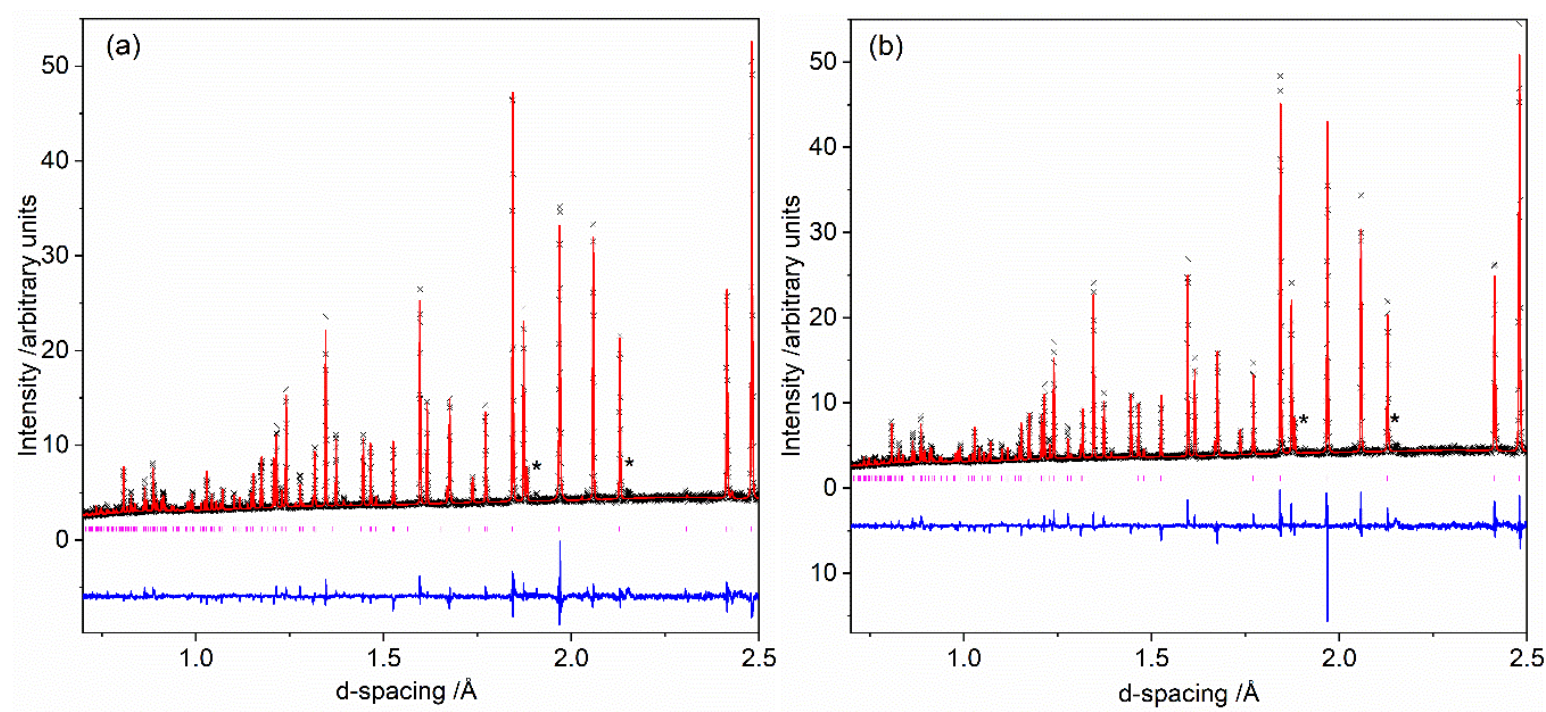

Fig. 2. Portions of Rietveld refinement profiles of $\mathrm{RbNbW}_{2} \mathrm{O}_{9} \mathrm{PND}$ data at a) $673 \mathrm{~K}$ using the $P 6_{3} m c$ model and b) $723 \mathrm{~K}$ using the $P 6 \mathrm{~mm}$ model. The peaks marked '*' indicate peaks attributable to the vanadium can.

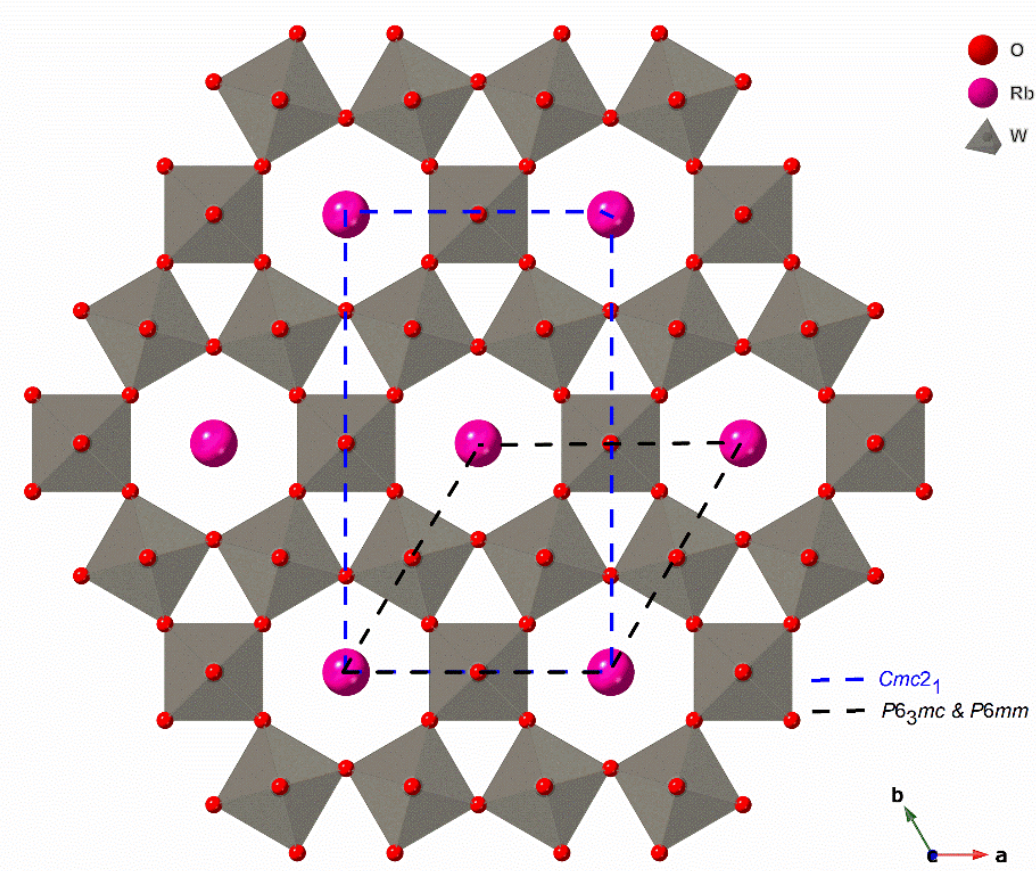

Fig. 3. Polyhedral view of the HTB structure viewed along the $c$-axis indicating the change in unit cell size between the orthorhombic (blue) and hexagonal space groups (black). 
Table 2. Crystallographic data for $\mathrm{RbNbW}_{2} \mathrm{O}_{9}$ at $673 \mathrm{~K}$ modelled in the $P 6_{3} m c$ space group $[a=7.3723$ (1), $c=7.8719$ (1) $\AA]$.

\begin{tabular}{llllll}
\hline Atom & $\begin{array}{l}\text { Wyckoff } \\
\text { Position }\end{array}$ & \multicolumn{1}{l}{$y$} & $z$ & $\begin{array}{l}100 \times U_{i s o} \\
\left(\AA^{2}\right)\end{array}$ \\
\hline $\mathrm{Rb} 1$ & $2 a$ & 0 & 0 & $0.2402(12)$ & $9.75(9)$ \\
$\mathrm{O} 1$ & $6 c$ & $0.5042(4)$ & $0.4958(4)$ & $0.2414(4)$ & $3.86(4)$ \\
$\mathrm{O} 2$ & $6 c$ & $0.7902(7)$ & $0.2098(7)$ & $0.9858(5)$ & $2.56(3)$ \\
$\mathrm{O} 3$ & $12 d$ & $0.7888(7)$ & $0.2112(7)$ & $0.5010(5)$ & $2.54(3)$ \\
$\mathrm{Nb} / \mathrm{W} 1$ & $6 c$ & $0.4982(6)$ & $0.5018(6)$ & $0.0169(6)$ & $2.49(4)$ \\
\hline
\end{tabular}

Between 288 and $623 \mathrm{~K}$, the orthorhombic $C m c 2_{1}$ space group provides the best fit to the diffraction data. The convergence of $a$ - and $b$ - lattice parameters suggest a gradual decrease in the degree of orthorhombicity with increasing temperature until adoption of the hexagonal $P 6_{3} m c$ space group at $673 \mathrm{~K}$.

Above $673 \mathrm{~K}$, peaks corresponding to $c$-axis doubling are absent (Fig. 1b) indicating the loss of octahedral tilting. From the group-subgroup relationship transition from $P 6_{3} m c\left(a \sim a_{0}, c \sim\right.$ $\left.2 c_{0}\right)$ may only proceed via supergroups $P 6_{3} / m m c\left(a \sim a_{0}, c \sim 2 c_{0}\right)$ or P6mm $\left(a \sim a_{0}, c \sim c_{0}\right)$. As this transition corresponds to loss of octahedral tilting, only $P 6 \mathrm{~mm}$ remains as a possibility due to the doubled $c$-axis present in the $P 6_{3} / m m c$ model. A similar transition to this is observed with the loss of tilting in the related $\mathrm{CsNbW}_{2} \mathrm{O}_{9}$ system.[9] Rietveld refinement of diffraction data between 723 and $973 \mathrm{~K}$ indicated a good fit using the $P 6 \mathrm{~mm}$ model. The paraelectric $P 6 / \mathrm{mmm}$ model was also tested in this temperature range but the fits were of an inferior quality (Table S1). Moreover, due to the absence of an abrupt change in both the lattice parameters and dielectric data, the polar $P 6 \mathrm{~mm}$ model remains the most likely. Crystallographic data for the $P 6 \mathrm{~mm}$ phase at $723 \mathrm{~K}$ is presented in Table 3 and the corresponding Rietveld fit in Fig. $2 \mathrm{~b}$. 
Table 3. Crystallographic data for $\mathrm{RbNbW}_{2} \mathrm{O}_{9}$ at $723 \mathrm{~K}$ modelled in the $P 6 m m$ space group $[a=7.3750$ (1), $c=3.9375$ (1) $\AA]$.

\begin{tabular}{llllll}
\hline Atom & $\begin{array}{l}\text { Wyckoff } \\
\text { Position }\end{array}$ & $x$ & $y$ & $z$ & $\begin{array}{l}100 \times U_{\text {iso }} \\
\left(\AA^{2}\right)\end{array}$ \\
\hline $\mathrm{Rb} 1$ & $1 a$ & 0 & 0 & $0.5122(28)$ & $10.22(9)$ \\
$\mathrm{O} 1$ & $3 c$ & 0.5 & 0 & $0.5266(8)$ & $4.13(5)$ \\
$\mathrm{O} 2$ & $6 e$ & $0.7896(1)$ & $0.2104(1)$ & $0.0230(7)$ & $2.75(3)$ \\
$\mathrm{Nb} / \mathrm{W} 1$ & $3 c$ & 0.5 & 0 & $0.9727(6)$ & $2.50(4)$ \\
\hline
\end{tabular}

Above $973 \mathrm{~K}$, a decrease in the $c$-axis parameter is observed like that previously observed in $\mathrm{CsNbW}_{2} \mathrm{O}_{9}$.[9] While this is suggestive of a structural transition to the paraelectric $(P 6 / \mathrm{mmm})$ phase, all refinements above $973 \mathrm{~K}$ are still a slightly better fit in the polar $86 \mathrm{~mm}$ model (Table $\mathrm{S} 1$ and Figure S5) suggesting that the transition to $P 6 / \mathrm{mmm}\left(T_{\mathrm{C}}\right)$ occurs above the available data range. Evolution of the lattice parameters versus temperature based on the Cmc2 1 (288$623 \mathrm{~K}), P 6{ }_{3} m c(673 \mathrm{~K}), P 6 m m(723-973 \mathrm{~K})$ and $P 6 / m m m(>973 \mathrm{~K})$ phases is shown in Fig. 4. Finer temperature increments would be necessary to establish more precise points for both the transition between $C m c 2_{1}$ and $P 6_{3} m c$ and the subsequent $P 6_{3} m c$ to $P 6 m m$ transition.

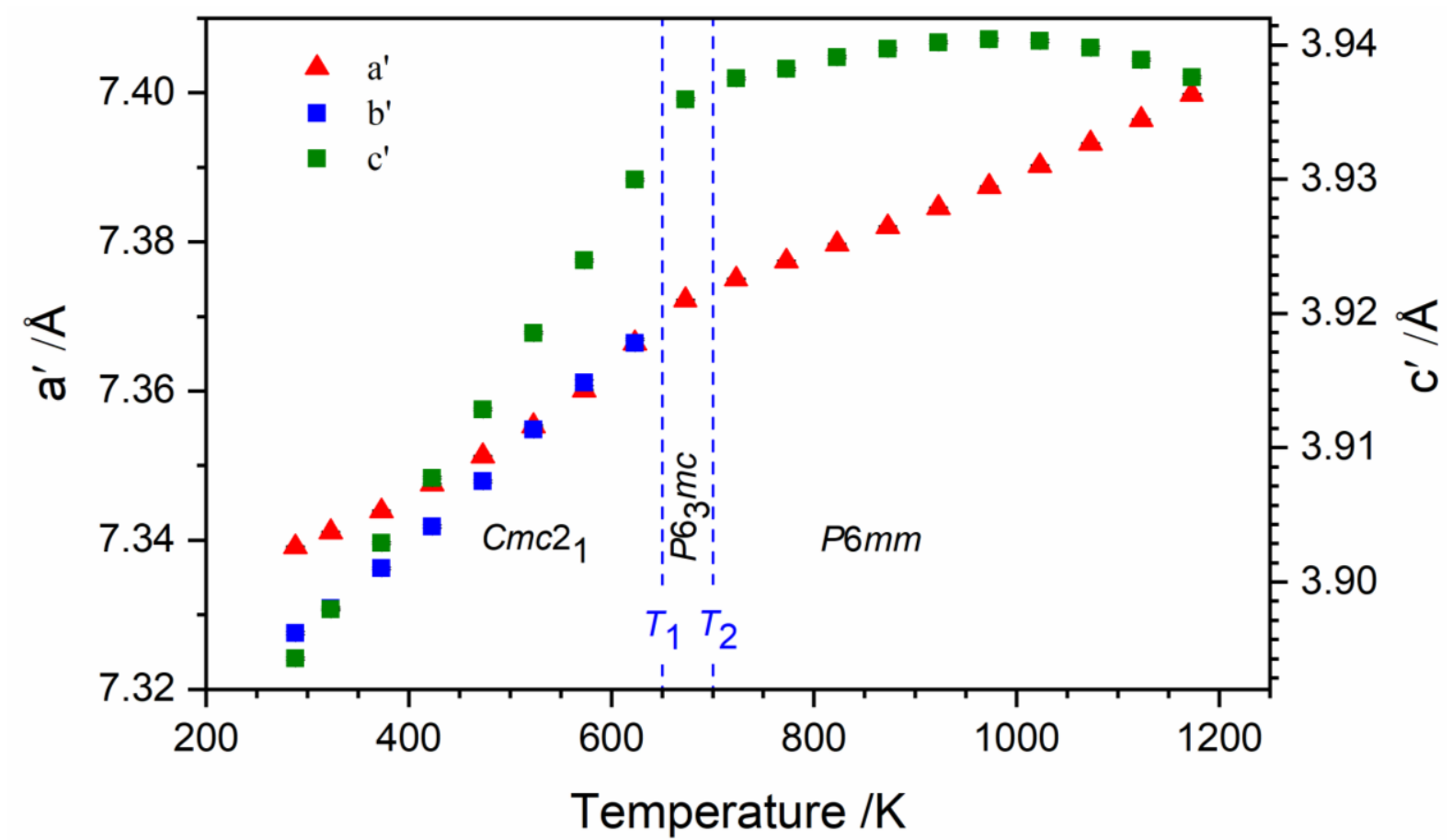

Fig. 4. Lattice parameters obtained from Rietveld refinement of PND data in the assigned space groups. All cell dimensions have been reduced to the $P 6 / \mathrm{mmm}$ aristotype which has $a^{\prime}=a_{0}$ and $c^{\prime}=c_{0}: P 6 \mathrm{~mm}$ $a^{\prime}=a_{0}, c^{\prime}=c_{0} ; P 6_{3} m c a^{\prime}=a_{0}, c^{\prime}=c / 2 ; C m c 2_{1} a^{\prime}=a_{0}, b^{\prime}=b_{0} / \sqrt{ } 3, c^{\prime}=c_{0} / 2$. 


\subsection{Discussion}

The crystal structure of the ambient temperature phase, $C m c 2_{1}$, of $\mathrm{RbNbW}_{2} \mathrm{O}_{9}$ has been established,[10] however, the two higher temperature $\left(P 6_{3} m c\right.$ and $\left.P 6 \mathrm{~mm}\right)$ phases, have not been reported previously. The general trends observed in tilting behaviour with increasing temperature for $\mathrm{RbNbW}_{2} \mathrm{O}_{9}$ are similar to those observed in $\mathrm{CsNbW}_{2} \mathrm{O}_{9}$, but the absence of an additional cell-tripling $\left(\mathrm{K}_{3}\right)$ mode in the $\mathrm{Rb}$-compound indicates a fundamental difference between the two systems. The thermal evolution of the $B-\mathrm{O}$ bond lengths in the $B 1 \mathrm{O}_{6}$ octahedra for $\mathrm{RbNbW}_{2} \mathrm{O}_{9}$ is shown in Fig. 5. This clearly shows a gradual reduction in the degree of second-order Jahn-Teller (SOJT) distortion on the axial $B-\mathrm{O} 1$ bonds as the loss of the orthorhombic distortion and transition from $C m c 2_{1}$ to $P 6_{3} m c$ is approached. Although there was no clear evidence for a first-order transition in either the lattice parameters or dielectric data there is a slight step-change in the data at $T_{1}$ supporting the discontinuous nature of the $C m c 2_{1}$ to $P 6_{3} m c$ transition. Convergence of the $B 1-\mathrm{O} 4$ and $B 1-\mathrm{O} 6$ bond lengths is observed above $673 \mathrm{~K}$, corresponding to the loss of octahedral tilting and the transformation from $P 6_{3} m c$ to $P 6 \mathrm{~mm}$. Selected bond lengths and bond angles for the $P 6 \mathrm{~mm}$ phase at $723 \mathrm{~K}$ are given in Table 4 and the distortion of the $\mathrm{BO}_{6}$ octahedron illustrated in Fig. 6 highlighting the continued presence of the SOJT distortion after loss of octahedral tilting.
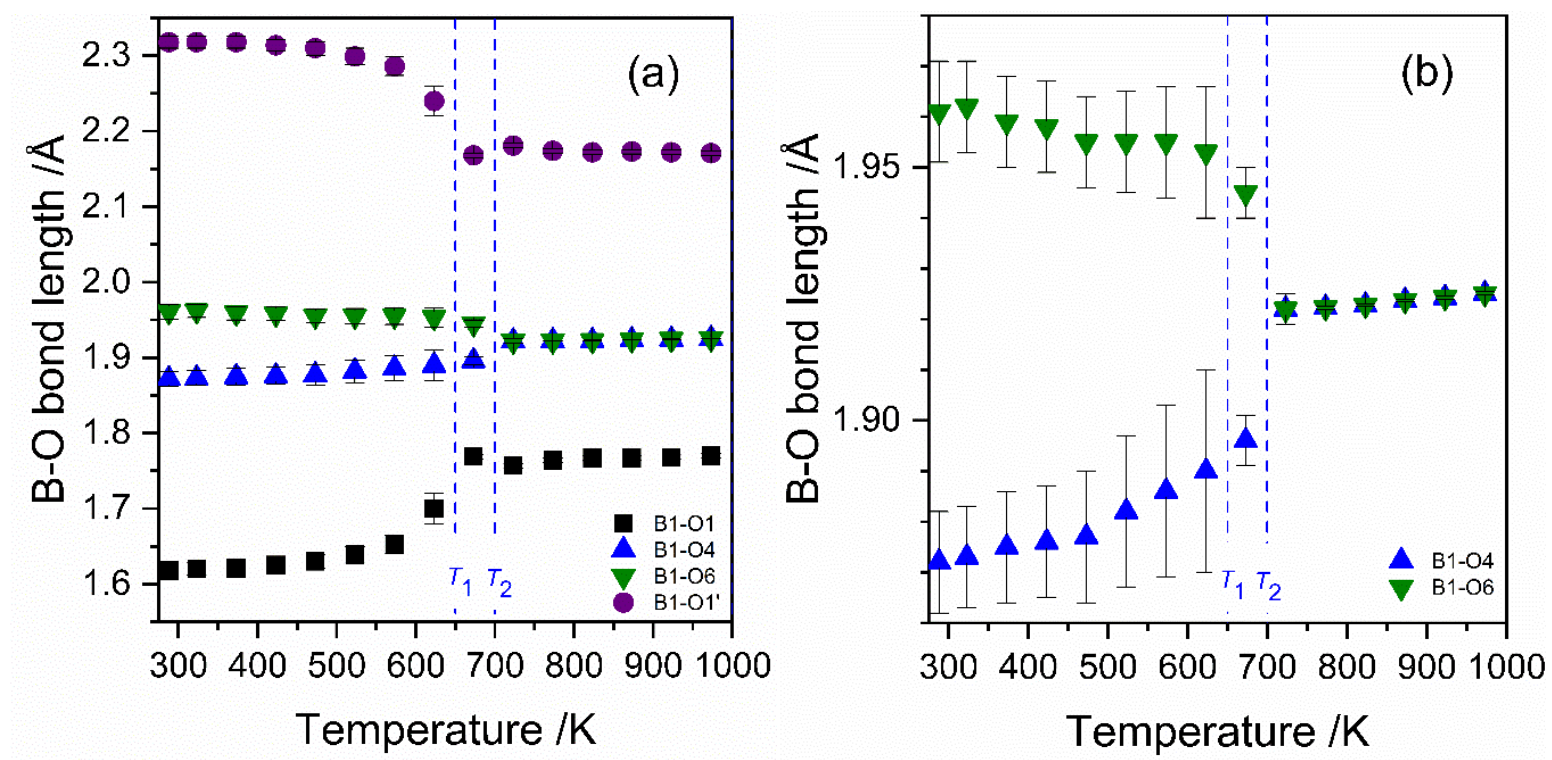

Fig. 5. Thermal evolution of $B 1-\mathrm{O}$ bond lengths over the region 288 to $973 \mathrm{~K}$, showing a) a decrease in the axially second-order Jahn-Teller distorted $B 1-\mathrm{O} 1$ bonds as the $C m c 2_{1}$ to $P 6_{3} m c$ phase transition is approached and $\mathrm{b}$ ) the convergence of the $B 1-\mathrm{O} 4$ and $B 1-\mathrm{O} 6$ bond lengths with the loss of octahedral tilting. 
Table 4. Bond lengths and selected bond angles of the $P 6 \mathrm{~mm}$ phase of $\mathrm{RbNbW}_{2} \mathrm{O}_{9}$ at $723 \mathrm{~K}$.

\begin{tabular}{llllll}
\hline $\mathrm{Rb}-\mathrm{O}$ & Bond length $(\AA)$ & $B-\mathrm{O}$ & Bond length $(\AA)$ & $\mathrm{O}-B-\mathrm{O}$ & Bond angle $\left({ }^{\circ}\right)$ \\
\hline $\mathrm{Rb}-\mathrm{O} 1$ & $3.6879(2) \times 6$ & $B-\mathrm{O} 1$ & $2.181(3)$ & $\mathrm{O} 1-B-\mathrm{O} 2$ & $84.09(8) \times 4$ \\
$\mathrm{Rb}-\mathrm{O} 2$ & $3.307(7) \times 6$ & $B-\mathrm{O} 1^{\prime}$ & $1.757(3)$ & $\mathrm{O} 1^{\prime}-B-\mathrm{O} 2$ & $95.91(8) \times 4$ \\
$\mathrm{Rb}-\mathrm{O} 2$ & $3.357(7) \times 6$ & $B-\mathrm{O} 2$ & $1.9220(3) \times 4$ & $\mathrm{O} 2-B-\mathrm{O} 2$ & $90.03(4) \times 2$ \\
& & & & $\mathrm{O} 2-B-\mathrm{O} 2$ & $88.75(4) \times 2$ \\
\hline
\end{tabular}

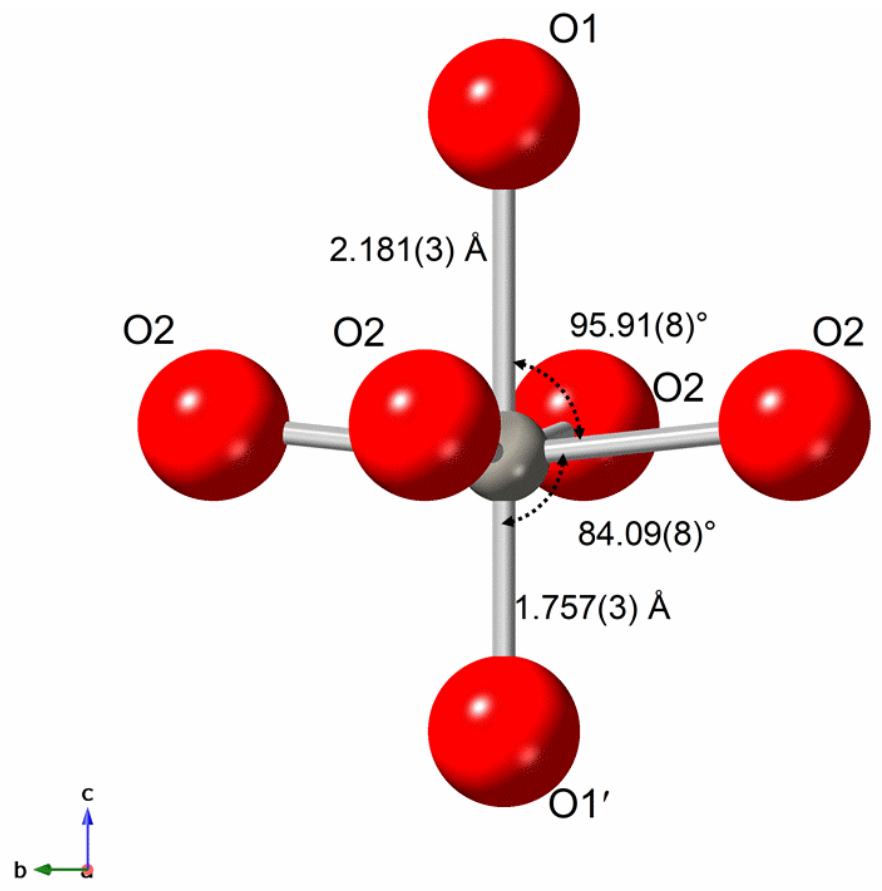

Fig. 6. Ball and stick representation of the $B_{0}$ octahedron in the $P 6 m m$ phase of $\mathrm{RbNbW}_{2} \mathrm{O}_{9}$ at $723 \mathrm{~K}$ highlighting the SOJT distortion of the $B-\mathrm{O} 1$ bonds (difference between $B-\mathrm{O} 1$ and $B-\mathrm{O} 1{ }^{\prime}$ bond lengths $\sim 0.424 \AA$ ).

To further probe the nature of each phase transition, the magnitudes of the relevant distortion modes associated with each phase were examined using ISODISTORT [13] as a function of temperature, Fig. 6. The displacive $\Gamma_{2}^{-}$mode is responsible for the adoption of a polar space group and has a relatively constant magnitude at all temperatures below $1000 \mathrm{~K}$. However, there is some evidence of a slight decrease in $\Gamma_{2}^{-}$at the highest temperatures studied which corresponds to a reduction in the polarity of the material as $T_{\mathrm{C}}$ is approached. This slight decrease is likely responsible for the observed contraction of the $c$-axis. The $\mathrm{A}_{3}{ }^{+}$mode exhibits a step change at the $\sim 650 \mathrm{~K}$ transition, correlating with the transition from an orthorhombic to hexagonal structure while retaining octahedral tilting. Both $\mathrm{A}_{6}{ }^{+}$and $\Gamma_{5}{ }^{-}$(a coupled mode arising 
from the introduction of $\mathrm{A}_{6}^{+}$) show a gradual trend towards zero with the transition from $\mathrm{Cmc} 2_{1}$ to $P 6_{3} m c$ corresponding to the loss of orthorhombicity. Extrapolation of the trends observed for both distortion modes was carried out using a weighted power law (mode amplitude $=\mathrm{A}\left(T_{1}\right.$ $T)^{\beta}$ ) to clarify the transition temperature corresponding to the loss of the orthorhombic distortion $\left(T_{1}\right)$. The critical exponent for both $\Gamma_{5}{ }^{-}$and $\mathrm{A}_{6}{ }^{+}$was $\beta=0.296$ with a fit constant $\mathrm{A}$ $=0.13$ and 0.051 , respectively. The values calculated for $T_{1}$ indicate a transition temperature of $\sim 655$ and $630 \mathrm{~K}$ for $\mathrm{A}_{6}{ }^{+}$and $\Gamma_{5}{ }^{-}$, respectively. While transition temperatures for both distortion modes are expected to be the same there is a slight difference between the those calculated, this is likely due to the quality of the fit of each curve. These values do remain in good agreement with both the change observed in the lattice parameters and the $B 1-\mathrm{O}$ bond lengths. As a similar extrapolation of the data cannot be carried out for the loss of octahedral tilting $\left(T_{2}\right)$,the transition sequence is summarised as:

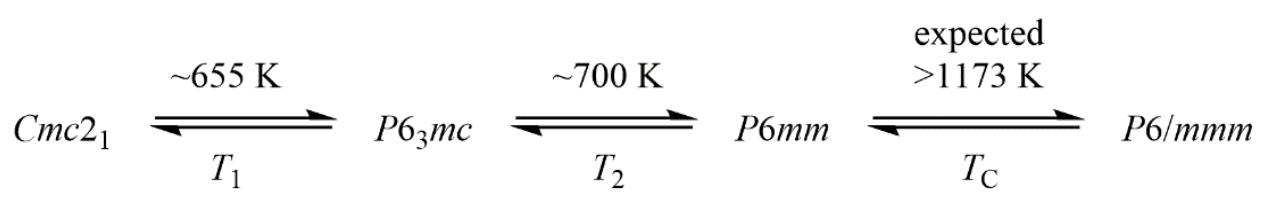

Measurements at finer temperature increments would be beneficial in determination of the exact transition temperatures and critical exponents.

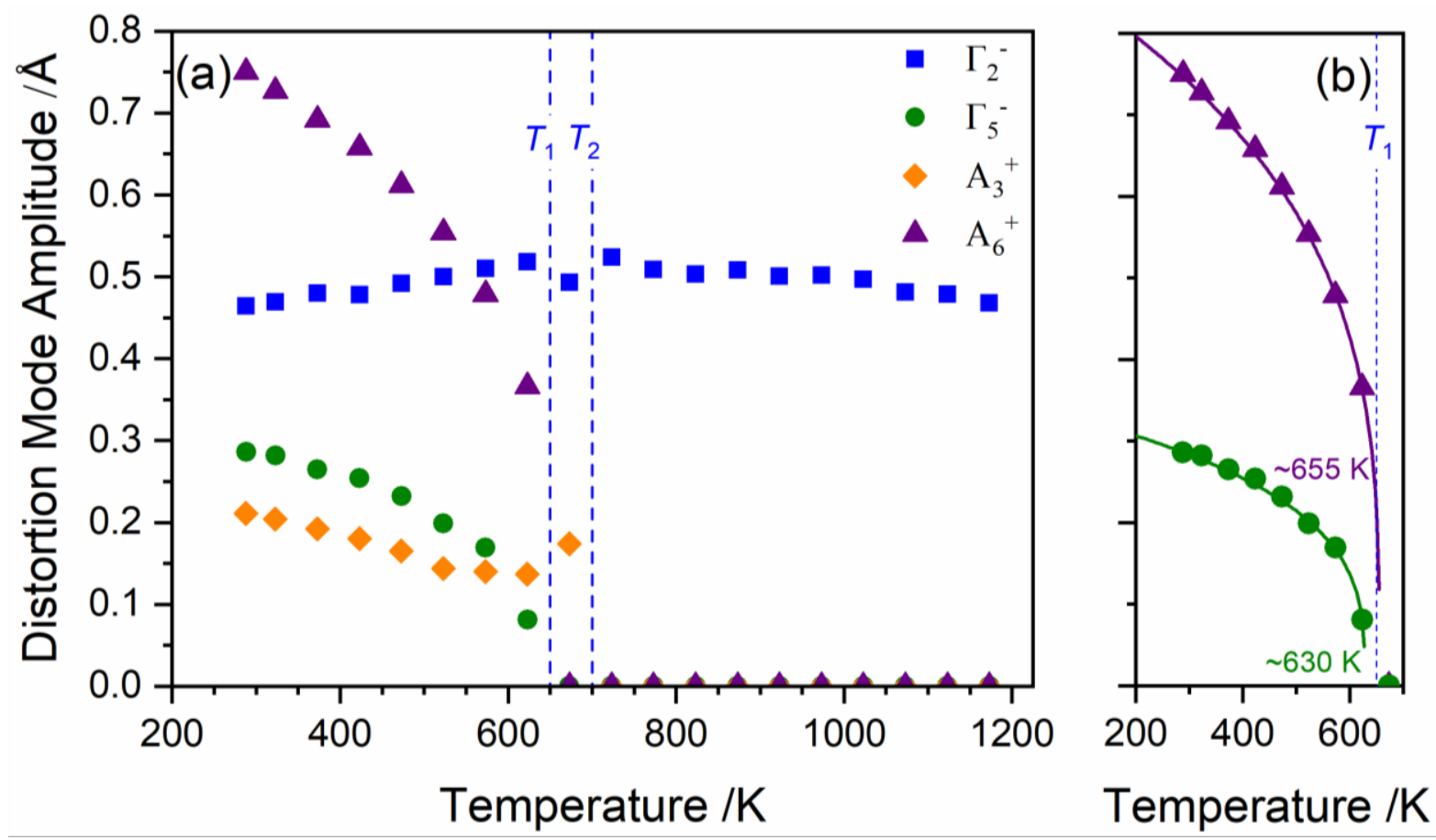

Fig. 6. a) Amplitudes of the distortion modes calculated from refined Rietveld models using ISODISTORT. b) Curve-fitting of the $\mathrm{A}_{6}{ }^{+}$mode and $\Gamma_{5}{ }^{-}$(a coupled mode arising from introduction of $\mathrm{A}_{6}{ }^{+}$). 


\section{Summary and conclusions}

The thermal evolution of the phase behaviour and crystal structure of the polar hexagonal tungsten bronze $\mathrm{RbNbW}_{2} \mathrm{O}_{9}$ has been characterised using powder neutron diffraction supported by symmetry-mode analysis and dielectric measurements.

The phase transition sequence in $\mathrm{RbNbW}_{2} \mathrm{O}_{9}$ has been shown to be driven by loss of an octahedral tilting mode $\left(\mathrm{A}_{6}{ }^{+}\right)$followed by the loss of a second octahedral tilting mode $\left(\mathrm{A}_{3}{ }^{+}\right)$ with increasing temperature. This is expected to be followed by the loss of polarity, corresponding to the loss of a displacive $\Gamma_{2}^{-}$mode, however this appears to occur above the temperature range studied here. While a similar phase transition sequence has previously been observed for the analogous Cs composition, the absence of a $\mathrm{K}_{3}$ mode responsible for unit celltripling (and ultimately a symmetry-breaking mechanism resulting in improper ferroelectricity) results in a different space group assignment. The absence of this distortion mode in $\mathrm{RbNbW}_{2} \mathrm{O}_{9}$ results in a symmetry-breaking mechanism resulting in proper ferroelectricity and is likely driven by the smaller $\mathrm{Rb}^{+}$cation $\left[r\left(M^{+}\right)=1.72 \AA\right]$ providing less stabilisation of the regularity of the hexagonal A-site channels compared with the larger $\mathrm{Cs}^{+}$cation $\left[r\left(M^{+}\right)=1.88\right.$ $\AA$ ]. Quoted ionic radii are for 12-fold coordination.[17] This significantly different phase transition sequence between the related $\mathrm{RbNbW}_{2} \mathrm{O}_{9}$ and $\mathrm{CsNbW}_{2} \mathrm{O}_{9}$ compositions highlights the importance of using high-resolution powder neutron diffraction and detailed symmetrymode analysis in structural determination of materials of this type.

We thank the Science and Technology Facilities Council (STFC) for the provision of neutron diffraction facilities at ISIS (HRPD experiment RB1710021, doi: 10.5286/ISIS.E.RB1710021) and the School of Chemistry, University of St Andrews for funding of a studentship to JAM through the EPSRC doctoral training grant (grant No. EP/K503162/1). The research data supporting this publication can be accessed at (https://doi.org/10.17630/0e237288-4392-4cd0b4d9-47409c824dac).

\section{Conflict of Interest}

The authors declare no conflict of interest.

\section{Author Contributions}


JAM collected and analysed the diffraction data with input from ASG, PL and FDM. Electrical data were collected and analysed by JAM under the supervision of FDM. FDM conceived and oversaw the study. All authors contributed to the writing of the manuscript.

\section{References}

[1] P.S. Halasyamani, K. Poeppelmeier, Noncentrosymmetric Oxides, Chem. Mater. 10 (1998) 2753-2769. doi:10.1021/cm980140w.

[2] R.J.D. Tilley, Perovskites: Structure-Property Relationships, Wiley-Blackwell, Oxford, 2016.

[3] A.R. Chakhmouradian, P.M. Woodward, Celebrating 175 years of perovskite research: a tribute to Roger H. Mitchell, Phys. Chem. Miner. 41 (2014) 387-391. doi:10.1007/s00269-014-0678-9.

[4] J. Simon, A. Ravez, Solid-state chemistry and non-linear properties of tetragonal tungsten bronzes materials, C. R. Chim. 9 (2006) 1268-1276. doi:10.1016/j.crci.2006.04.001.

[5] X. Zhu, M. Fu, M.C. Stennett, P.M. Vilarinho, I. Levin, C.A. Randall, J. Gardner, F.D. Morrison, I.M. Reaney, A crystal-chemical framework for relaxor versus normal ferroelectric behavior in tetragonal tungsten bronzes, Chem. Mater. 27 (2015) 32503261. doi:10.1021/acs.chemmater.5b00072.

[6] A. Magnéli, A.I. Virtanen, J. Olsen, A.I. Virtanen, N.A. Sörensen, Studies on the hexagonal tungsten bronzes of potassium, rubidium, and cesium., Acta Chem. Scand. 7 (1953) 315-324. doi:10.3891/acta.chem.scand.07-0315.

[7] V.A. Isupov, Some crystal chemical aspects of ferroelectrics with structure of the hexagonal tungsten bronze type, Ferroelectrics. 211 (1998) 209-232. doi:10.1080/00150199808232344.

[8] H.Y. Chang, T. Sivakumar, K.M. Ok, P.S. Halasyamani, Polar hexagonal tungsten bronze-type oxides: KNbW2O9, RbNbW2O9 and KTaW2O9, Inorg. Chem. 47 (2008) 8511-8517. doi:10.1021/ic800573k.

[9] J.A. McNulty, T.T. Tran, P.S. Halasyamani, S.J. McCartan, I. MacLaren, A.S. Gibbs, F.J.Y. Lim, P.W. Turner, J.M. Gregg, P. Lightfoot, F.D. Morrison, An electronically driven improper ferroelectric: tungsten bronzes as microstructural analogs for the 
hexagonal manganites, Adv. Mater. 31 (2019) 1903620. doi:10.1002/adma.201903620.

[10] J.A. McNulty, A.S. Gibbs, P. Lightfoot, F.D. Morrison, Octahedral tilting in the polar hexagonal tungsten bronzes RbNbW2O9 and KNbW2O9, Acta Cryst. B75 (2019) 815-821. doi:10.1107/S2052520619009260.

[11] A.C. Larson, R.B. Von Dreele, General Structure Analysis System (GSAS), (2004).

[12] B.H. Toby, EXPGUI, a graphical user interface for GSAS, J. Appl. Crystallogr. 34 (2001) 210-213. doi:10.1107/S0021889801002242.

[13] B.J. Campbell, H.T. Stokes, D.E. Tanner, D.M. Hatch, ISODISPLACE: A web-based tool for exploring structural distortions, J. Appl. Crystallogr. 39 (2006) 607-614. doi:10.1107/S0021889806014075.

[14] T.A. Whittle, S. Schmid, C.J. Howard, Octahedral tilting in the tungsten bronzes, Acta Cryst. B71 (2015) 342-348. doi:10.1107/S2052520615008252.

[15] B. Campbell, C.J. Howard, T.B. Averett, T.A. Whittle, S. Schmid, S. Machlus, C. Yost, H.T. Stokes, An algebraic approach to cooperative rotations in networks of interconnected rigid units, Acta Cryst. A74 (2018) 408-424. doi:10.1107/S2053273318009713.

[16] T.A. Whittle, S. Schmid, C.J. Howard, Octahedral tilting in the tungsten bronzes. Addendum., Acta Cryst. B74 (2018) 742-744. doi:10.1107/S2052520618015263.

[17] R.D. Shannon, Revised effective ionic radii and systematic studies of interatomic distances in halides and chalcogenides, Acta. Cryst. A32 (1976) 751-767. doi:10.1107/S0567739476001551. 